\title{
Consideraciones de la Administración sobre la aplicación de la reglamentación a edificios existentes
}

\author{
Public Administration considerations about the \\ implementation of regulations in existing buildings
}

L. Vega ${ }^{(*)}$, J.L. Posada ${ }^{(*)}$

RESUMEN

La aplicación de la reglamentación en el ámbito de la edificación existente es una problemática difícil, que preocupa a todos los técnicos. La pregunta de si es posible alcanzar los niveles de prestación demandados en la actualidad y recogidos en la reglamentación para obra nueva cuando se opera sobre edificios existentes, salvaguardando al mismo tiempo la seguridad jurídica de los diferentes agentes, es un problema que requiere de un amplio debate.

El objetivo de este artículo es explicar las dificultades para dar criterios en las intervenciones en la edificación existente y las estrategias que está valorando la Administración.

\section{SUMMARY}

The compliance with building regulations in existing buildings is a difficult problem. The question about if it is possible to fulfil the performance levels required by the society and stated in new buildings regulations when operating on existing ones, while safeguarding legal responsibilities of participants in the process opens an interesting debate.

The aim of this paper is to explain the difficulties found on giving criteria for these cases and the strategies that the public administration is considering.
Palabras clave: Rehabilitación; reglamentación; prestaciones; cambios de uso; reformas; ampliaciones.
Keywords: Renovation; regulations; performances; change of occupancy; alterations; additions. 


\section{APLICACIÓN DE LA REGLAMENTA- CIÓN A EDIFICIOS EXISTENTES}

En rehabilitación, se opera sobre edificios existentes, en los cuales las características propias del edificio condicionan de forma considerable las posibles intervenciones, y donde algunas de las mismas pueden tener efectos contradictorios: si intentamos mejorar algunas prestaciones o características, podemos empeorar simultáneamente otras. El intentar alcanzar determinados niveles de prestación, análogos a los contemplados en los Documentos Básicos del Código Técnico de la Edificación(Real Decreto 314/2006), puede suponer impactos económicos y sociales inadmisibles, e incluso incompatibilidad con los niveles de protección del edificio.

El mismo CTE indica: "... el CTE se aplicará a las obras de ampliación, modificación, reforma o rehabilitación que se realicen en edificios existentes, siempre y cuando dichas obras sean compatibles con la naturaleza de la intervención y, en su caso, con el grado de protección que puedan tener los edificios afectados. La posible incompatibilidad de aplicación deberá justificarse en el proyecto $y$, en su caso, compensarse con medidas alternativas que sean técnica y económicamente viables".

Recientemente en el Documento Básico de seguridad de utilización y accesibilidad, DB SUA (Real Decreto 173/2010) se ha incorporado el siguiente párrafo:

"Cuando la aplicación de las condiciones de este DB en obras en edificios existentes no sea técnica o económicamente viable o, en su caso, sea incompatible con su grado de protección, se podrán aplicar aquellas soluciones alternativas que permitan la mayor adecuación posible a dichas condiciones. En la documentación final de la obra deberá quedar constancia de aquellas limitaciones al uso del edificio que puedan ser necesarias como consecuencia del grado final de adecuación alcanzado y que deban ser tenidas en cuenta por los titulares de las actividades."

Éste último párrafo, más evolucionado que el anterior, reconoce, por una parte, la imposibilidad en algunos casos de alcanzar el nivel exigencial del CTE con medidas alternativas, por ello se habla de "la mayor adecuación posible" y no de "compensación". Por otra parte, se establece la necesidad de dejar constancia del nivel de prestación alcanzado.

Ya es práctica habitual en la evaluación de estructuras existentes y así se reconoce en el Anejo D, evaluación estructural de edificios existentes del Documento Básico de Seguridad Estructural DB SE que "No es adecuada la utilización directa de las normas y reglas establecidas en este CTE en la evaluación estructural de edificios existentes, construidos en base a reglas anteriores a las actuales para los edificios de nueva construcción, ..."

En este sentido ya se han iniciado una serie actuaciones, tanto por parte del Ministerio como de otros agentes que demuestran la preocupación por este tema. Dichas iniciativas deberían tener un tratamiento homogéneo, pues en todos estos casos el problema es análogo: cómo establecer niveles de adecuación al CTE racionales para las distintas intervenciones que puedan darse en edificiación existente cuando existen condiciones específicas que impiden alcanzar los niveles establecidos por el CTE, pensados para obra nueva.

Por ello, el objetivo en rehabilitación debiera ser "mejorar" las condiciones del edificio de forma racional y coherente con las características iniciales del mismo, para adecuarlo en la medida de lo posible a las necesidades del usuario (individuales y colectivas) demandadas en la actualidad y establecidas en el CTE, sin menoscabar en cualquier caso las condiciones preexistentes o unos niveles mínimos que se consideren seguros.

En este tipo de intervenciones no parece posible establecer una exigencia de carácter universal, ya que de las características específicas de cada intervención se derivará un determinado nivel de adecuación razonable, técnica y económicamente viable, y respetuoso con el valor arquitectónico y patrimonial del edificio (véase Figura 1).

\section{Seguridad y accesibilidad frente a habitabilidad}

Si bien el objetivo a alcanzar en edificación existente debe ser homogéneo para todo el CTE, el enfoque, en general, es distinto cuando hablamos de seguridad que cuando hablamos de habitabilidad.

En habitabilidad parece razonable pensar únicamente en mejorar dentro de ajustes razonables el comportamiento del edificio. Cuando hablamos de Seguridad y de Accesibilidad, sin embargo, además hay, por una parte, ciertos mínimos que no se pueden soslayar porque afectan a la seguridad de las personas (lo que podríamos llamar riesgo asumido socialmente) $y$, por otra parte, derechos universales mínimos que también deben respetarse. 


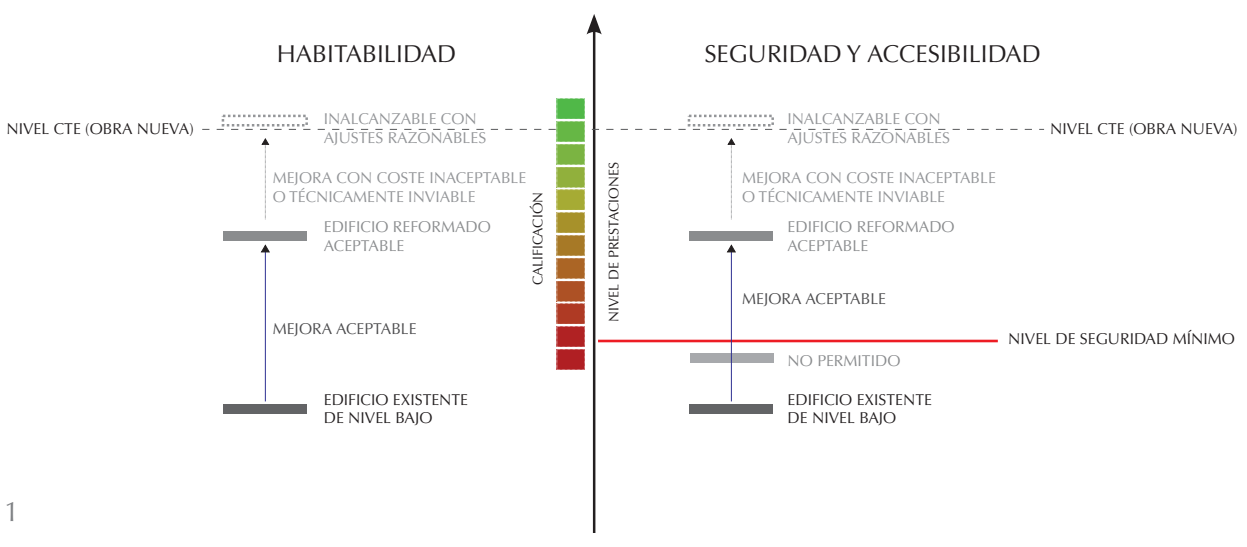

2. MAYOR ADECUACIÓN POSIBLE Y PROPORCIONALIDAD AL GRADO DE LA INTERVENCIÓN

Las características del entorno sobre el que se interviene son muy diversas. Incluyen desde edificios muy protegidos donde las condiciones para la intervención están muy limitadas hasta edificios donde la aplicación del CTE no presenta graves problemas como la obra nueva, con toda una serie de situaciones intermedias posibles (véase Figura 2).

Si se plantease como solución reducir los niveles de exigencia actuales en intervenciones en edificios existentes no se soluciona- ría el problema. La falta de homogeneidad inherente a la edificación existente dificulta la definición de un nivel de exigencia único y universal. Un nivel de exigencia que puede resultar proporcionado en algunos casos, resultará inviable, por cuestiones técnicas, económicas o arquitectónicas en otros. Fijarlo en un nivel alto supondría dificultades insoslayables en algunos casos, pero fijarlo en la banda baja implicaría niveles de mejora escasos en otras tantas situaciones. Todo ello parece conducir a mantener como objetivo a perseguir los niveles prestacionales del CTE e introducir criterios de flexibilidad acordes con las características específicas del edificio y tipo de intervención cuando no se puedan alcanzar.

\begin{tabular}{|llrl|}
\hline + Obra nueva & CONDICIONES DE CONTORNO & \multicolumn{2}{c|}{ Edificación Existente - } \\
- Nueva urbanización & - Cambio de uso Rehabilitación - \\
- Casco consolidado & - Ampliación & Total / Parcial - \\
- Condiciones físicas terreno & - Reforma & Etc. - \\
\hline
\end{tabular}

\begin{tabular}{|c|c|c|}
\hline \multirow[t]{2}{*}{ Sin condiciones de contorno } & \multicolumn{2}{|c|}{ Condiciones de contorno impiden aplicar el nivel de prestaciones del DB } \\
\hline & HABITABILIDAD & SEGURIDAD + ACCESIBILIDAD \\
\hline $\begin{array}{l}\text { - Nivel de prestaciones } \\
\text { establecidas en los DBs. }\end{array}$ & $\begin{array}{l}\text { - Mejorar hasta donde se pueda } \\
\text { con ajustes razonables según } \\
\text { estimaciones de confort del usuario }\end{array}$ & $\begin{array}{l}\text { - Mejorar hasta donde se pueda } \\
\text { con ajustes razonables siempre } \\
\text { que se supere un mínimo fijado } \\
\text { por la Administración }\end{array}$ \\
\hline & $\begin{array}{l}\text { - Relacionado con: zonas climáticas, } \\
\text { acústicas, soluciones constructivas, } \\
\text { año construcción, etc. }\end{array}$ & $\begin{array}{l}\text { - Relacionado con: condiciones } \\
\text { geométricas, espaciales, etc. }\end{array}$ \\
\hline
\end{tabular}

\begin{tabular}{|l|}
\hline Viabilidad ECONÓMICA / TÉCNICA / FUNCIONAL \\
\hline \begin{tabular}{l} 
Anejo D. DB SE \\
"No es adecuada la utilización directa de las normas y reglas establecidas en este CTE en la \\
evaluación estructural de edificios existentes, construidos en base a reglas anteriores a las \\
actuales para los edificios de nueva construcción". \\
\hline Introducción. III. Criterios de Aplicación. DB SUA \\
Cuando la aplicación de las condiciones de este DB en obras en edificios existentes no sea \\
técnica o económicamente viable o, en su caso, sea incompatible con su grado de protección, se \\
podrán aplicar aquellas soluciones alternativas que permitan la mayor adecuación posible a \\
dichas condiciones. En la documentación final de la obra deberá quedar constancia de aquellas \\
limitaciones al uso del edificio que puedan ser necesarias como consecuencia del grado final de \\
adecuación alcanzado y que deban ser tenidas en cuenta por los titulares de las actividades.
\end{tabular} \\
\hline
\end{tabular}

\begin{tabular}{|l||l|}
\hline DBs / Solución Alternativa & DBs / Solución Alternativa \\
\cline { 3 - 3 } $\begin{array}{l}\text { - Al menos, el mismo nivel de } \\
\text { prestaciones que las establecidas } \\
\text { en los DBs. }\end{array}$ & $\begin{array}{l}\text { - Igual nivel de prestaciones que las establecidas en los DBs. } \\
\text { - Si no es posible, mejoras: } \\
\end{array}$ \\
- Declaración de prestaciones de la solución \\
- Límites de uso \\
- Medidas compensatorias
\end{tabular}

1. Concepto de mejora aceptable.

2. Actuación en función de las condiciones de contorno.
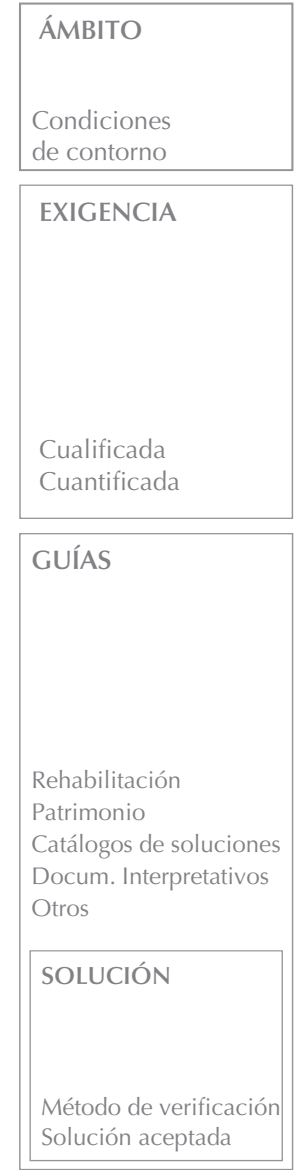
Es decir, establecer un doble sistema, que fije con carácter general perseguir el nivel del CTE (pero de forma proporcionada al nivel de intervención) para la generalidad de los casos, e introducir de forma explícita una cláusula de "salvaguarda" que permita al técnico, adecuar la mejora del edificio a las condiciones especificas del caso concreto.

Es necesario precisar y clasificar los diferentes tipos de intervención que pueden realizarse sobre edificios existentes, para poder definir con mayor criterio lo que conllevaría la aplicación del CTE en cada caso, diferenciando en qué casos o sobre qué elementos debe plantearse el objetivo de adaptación al CTE y en cuáles lo razonable (proporcionado a la intervención) es obligar exclusivamente a mantener el nivel de prestaciones preexistentes. Todo ello, sin menoscabo de que, obviamente, la intervención pueda plantear de forma voluntaria, la mejora de prestaciones sobre lo establecido de forma obligatoria, lo que podría fomentarse con una política de ayudas dirigida adecuadamente.

Por lo tanto, la mayor adecuación posible y el grado de proporcionalidad de la intervención deben definirse en función de:

- Las características específicas del edificio Uso, Valor patrimonial

- La posibilidad técnica, económica y funcional

- El tipo y nivel de intervención Reforma, ampliación, Cambio de uso

\section{ESTRUCTURA}

Asimismo, es importante destacar que el criterio o criterios específicos de aplicación en cada tipo de intervención, no tiene porque ser igual y homogéneo en todos los requisitos o exigencias. Sería necesario establecer en forma piramidal los criterios particulares, sobre la base de los definidos como más generales en escalones superiores (véase Figura 3).

Debe tenerse en cuenta la falta de homogeneidad actual de la estructura del CTE, derivada de la propia definición de requisitos básicos establecidos en la LOE. Para algunos requisitos, como Seguridad en caso de incendio, las exigencias constituyen un conjunto homogéneo que da respuesta a un único requisito, mientras que en otros casos, como Seguridad de utilización y accesibilidad o Salubridad, cada exigencia es en sí misma un requisito o sub-requisito.

La solución es incluir en la Parte I o, en su defecto, en la Introducción de cada DB de la Parte II, los criterios generales de aplicación para los diferentes tipos de intervención. A su vez, serán acompañados de criterios específicos de aplicación para cada requisito. Este esquema puede completarse con documentos con comentarios, guías, ejemplos resueltos, etc. que permitirán la particularización a cada exigencia, sub-exigencia, etc. en forma piramidal y jerárquica descendente.

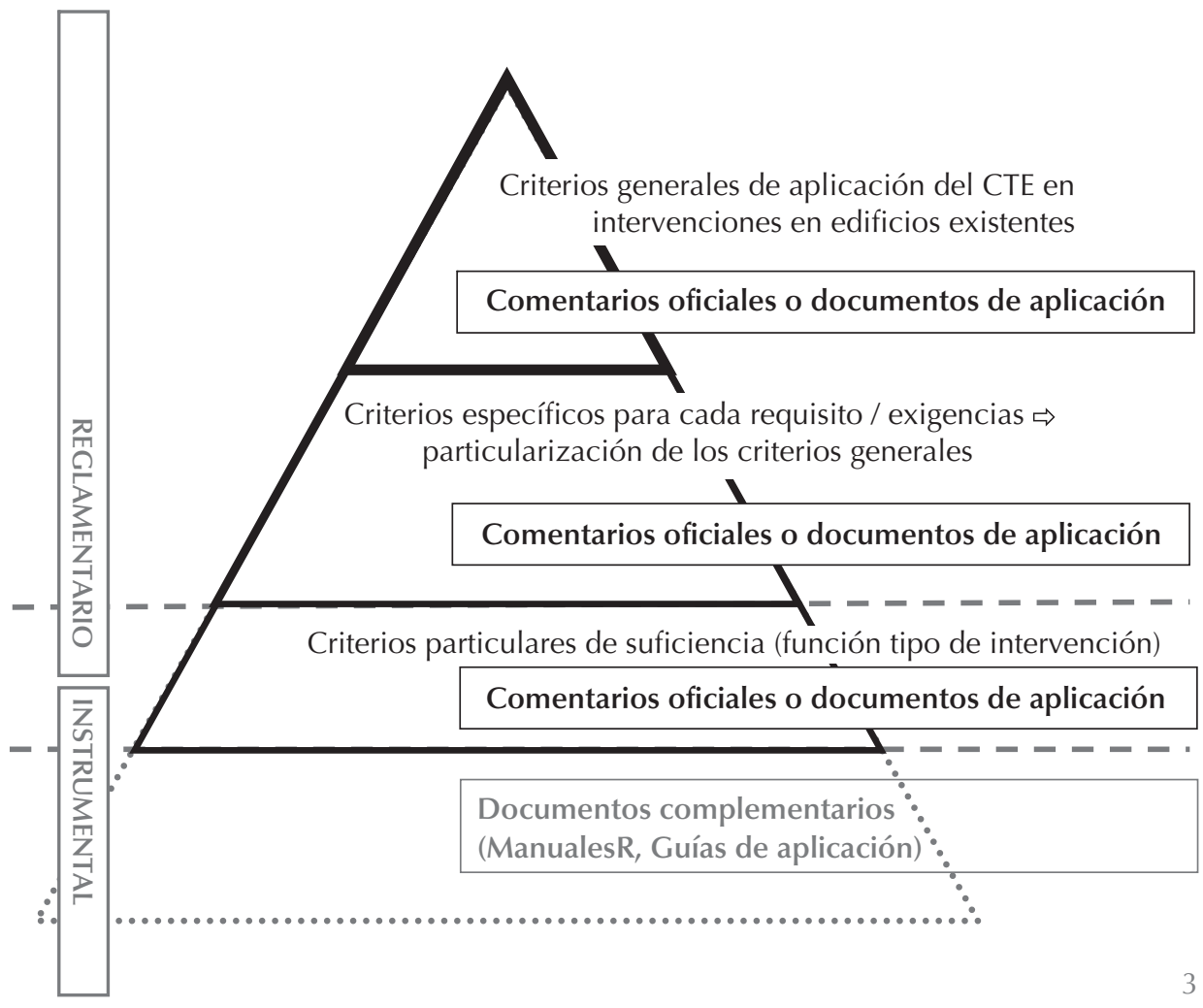




\section{TIPO DE INTERVENCIÓN}

Bajo el término de rehabilitación podrían englobarse un amplio conjunto de intervenciones cuyos objetivos, características y condicionantes son sensiblemente diferentes, siendo igualmente diferentes la forma en que debe entenderse la obligatoriedad de aplicación del CTE y de las diferentes exigencias básicas.

El CTE, en su Parte I y en muchos de los DB que conforman la Parte II, utiliza una serie de términos como ampliación, modificación, reforma o rehabilitación (Parte I, art. 2. (3)), que no se definen explícitamente y que se tratan de forma conjunta, sin matices ni diferenciación, en cuanto a la obligación de aplicación del CTE:

"Igualmente, el CTE se aplicará a las obras de ampliación, modificación, reforma o rehabilitación que se realicen en edificios existentes, siempre y cuando dichas obras sean compatibles con la naturaleza de la intervención y, en su caso, con el grado de protección que puedan tener los edificios afectados. La posible incompatibilidad de aplicación deberá justificarse en el proyecto $y$, en su caso, compensarse con medidas alternativas que sean técnica y económicamente viables".

Por lo que sería necesario:

- Sustituir el término rehabilitación (de concepción más global) por el de intervención en edificación existente, más riguroso y acorde con el conjunto de operaciones que dentro del mismo parecen englobarse.

- Definir el criterio general o criterios generales para la aplicación del CTE en al menos los tipos de intervención que podríamos considerar básicos: ampliación, reforma (posiblemente diferenciando distintos niveles de reforma), cambio de uso. El término modificación, incluido en CTE, Parte I, parece conceptualmente dentro de la reforma. Evidentemente se pueden dar combinaciones de ellos o intervenciones parciales, pero podrían extraerse los criterios aplicables en estos casos a partir de los anteriores.

- Concretar o particularizar los principios generales de aplicación a las diferentes exigencias básicas (o sub-exigencias, o sub-requisitos, según como quiera verse), quedando, no obstante, los criterios generales de aplicación como subsidiarios para aquellos casos no definidos explícitamente.

\section{CRITERIOS GENERALES DE APLICACIÓN DEL CTE A EDIFICIOS EXISTENTES}

Lo que se persigue es la mejora de los edificios, por lo que lo esencial, cuando se trata de intervenir en edificios existentes, es que no se reduzcan las condiciones de seguridad y habitabilidad que el edificio aporta antes de la intervención cuando sean menores a las que se exigen en la actualidad. En el caso de que estas condiciones existentes sean mayores, únicamente se podrán reducir hasta los niveles del CTE.

Parece lógico pensar que en ampliaciones la parte ampliada debe cumplir como si se tratara de una obra nueva. Esto puede conllevar además algunas adaptaciones de la parte existente cuando sea necesario para asegurar el cumplimiento de la ampliación, como es el caso, por ejemplo, de los recorridos de evacuación o itinerarios accesibles hasta la vía pública. No obstante hay que hacer algunos matices. En primer lugar, la separación entre la parte ampliada y la parte existente debe considerarse como parte de la ampliación y exigírsele todas las condiciones. Teniendo en cuenta que una ampliación supone un incremento del valor patrimonial del edificio parece natural que no se permita realizarla a menos que se consiga el cumplimiento total.

Respecto al cambio de uso es necesario cambiar el concepto que se ha manejado hasta el momento. Hasta ahora se entendía por cambio de uso, fórmula heredada de los documentos de protección contra incendios, al cambio entre alguno de los usos definidos para este requisito. Sin embargo, este enfoque del riesgo ligado al incendio no resulta adecuado cuando se introducen el resto de requisitos. En este nuevo enfoque debe entenderse por cambio de uso, respecto de un requisito aquel que modifica el perfil de uso (o riesgo) de dicho requisito, lo que implica que una misma intervención puede considerarse que altera el uso respecto de algunos requisitos y no respecto a otros.

Para cambios de uso, tratados individualmente para cada requisito como ya se ha comentado, en general también debe perseguirse el cumplimiento total del CTE. Sin embargo en este caso, se podrían admitir soluciones que no alcancen el umbral del CTE cuando el cambio que se produce es a una situación menos exigente que la anterior.

Cuando hablamos de reformas, el objetivo debe ser la adecuación proporcional al nivel de intervención y la flexibilidad para alcanzar este objetivo debe ser mayor que en el resto de intervenciones. Por ejemplo, 
cuando la adaptación no sea técnica o económicamente viable o sea incompatible con el grado de protección puede buscarse el mayor grado de adecuación posible. Por ejemplo, está claro que los elementos o productos que se modifiquen sustancialmente, sustituyan a uno existente o se incorporen nuevos, deberían cumplir las prestaciones que aportan individualmente. Se entiende que cuando la contribución de ese elemento sea mínima o nula para alcanzar la prestación, no se le exija el cumplimiento. Por ejemplo, si se actúa sobre la parte opaca de una fachada, el incremento de aislamiento acústico que se se puede conseguir será siempre muy reducido, a menos que se mejore la ventana que es el elemento débil y por lo tanto el más influyente en el comportamiento global de la fachada.

El resto de elementos del edificio en general pueden mantenerse. Habrá lógicamente algunas excepciones, por ejemplo en los casos que la administración considere especialmente peligrosos, cuando se considere necesario por otros motivos, tales como sostenibilidad, accesibilidad u otros beneficios sociales tutelados por las administraciones, cuando la envergadura de la obra sea importante, etc.

Cuando no se puedan alcanzar los niveles de prestación establecidos en el CTE, por no ser técnica o económicamente viable, es importante que se deje constancia de ello, así como cuando esta falta de adecuación implica condicionantes de uso y mantenimiento que haya que tener en cuenta.

También será necesario considerar y validar aquellas soluciones tradicionales que hayan demostrado un comportamiento adecuado en situaciones determinadas frente a ciertas exigencias, avaladas por la práctica y que no reciben un tratamiento adecuado en los modelos de comportamiento desarrollados para otros tipos de soluciones. Por ejemplo, si un edifico no presenta humedades puede considerarse cumplida la prestación de protección frente a la humedad aunque no se haya resuelto constructivamente según el CTE.

\section{TRABAJOS FUTUROS}

Por el momento, este esquema descansa en criterios bastante generales que deberán interpretarse para cada caso concreto y por lo tanto se resolverán posiblemente en el acuerdo entre autoridades de control, proyectistas y otros agentes involucrados. La paulatina aplicación de estos criterios, la recopilación de casos y otros estudios que ya se están realizando deberían servir para desarrollar de forma más pormenorizada, aunque posiblemente siempre flexible, los límites de aplicación de la reglamentación, bien sea mediante la publicación de catálogos de casos concretos, bien sea mediante una definición más explicita de los parámetros de proporcionalidad y flexibilidad.

\section{AGRADECIMIENTOS}

Estas consideraciones son resultado del debate y análisis compartido con la Unidad de Calidad en la Construcción del Instituto Eduardo Torroja de Ciencias de la Construcción (IETCC), sin cuyas aportaciones no hubiera sido posible. De igual forma, debemos también reconocer la deuda con otros trabajos que han ayudado a estas reflexiones elaborados por el Instituto de Tecnología de la Construcción de Cataluña (ITeC), la Fundación DOCOMOMO y el Consejo Superior de Colegios de Arquitectos de España (CSCAE).

\section{BIBLIOGRAFÍA}

(1) Real Decreto 173/2010 de 19 de febrero, por el que se modifica el Código Técnico de la Edificación, aprobado por el Real Decreto 314/2006, de 17 de marzo, en materia de accesibilidad y no discriminación de las personas con discapacidad. $\mathrm{n}^{\circ} 61$ (11 de marzo 2010) pp. 24510 - 24562.

(2) Real Decreto 314/2006 de 17 de marzo, por el que se aprueba el Código Técnico de la Edificación. no 74 (28 de marzo 2006) pp. 11816 - 11831. 